Relations industrielles

Industrial Relations

\title{
Les nouvelles lois du travail et l'activité de grève
}

\section{Robert Lacroix et André Lespérance}

Volume 43, numéro 4, 1988

URI : https://id.erudit.org/iderudit/050452ar

DOI : https://doi.org/10.7202/050452ar

Aller au sommaire du numéro

Éditeur(s)

Département des relations industrielles de l'Université Laval

ISSN

0034-379X (imprimé)

1703-8138 (numérique)

Découvrir la revue

Citer cet article

Lacroix, R. \& Lespérance, A. (1988). Les nouvelles lois du travail et l'activité de grève. Relations industrielles / Industrial Relations, 43(4), 812-828.

https://doi.org/10.7202/050452ar
Résumé de l'article

Les auteurs évaluent l'effet de deux types de lois du travail sur l'activité de grève, à savoir celles visant à réduire telle activité et d'autres qui ne cherchent pas à la reduire mais qui peuvent l'affecter indirectement par leur incidence sur les rapports de force syndical-patronal.
Tous droits réservés @ C Département des relations industrielles de l'Universite Laval, 1988
Ce document est protégé par la loi sur le droit d'auteur. L’utilisation des services d'Érudit (y compris la reproduction) est assujettie à sa politique d'utilisation que vous pouvez consulter en ligne.

https://apropos.erudit.org/fr/usagers/politique-dutilisation/ 


\title{
Les nouvelles lois du travail et l'activité de grève
}

\author{
Robert Lacroix \\ et \\ André Lespérance
}

Les auteurs évaluent l'effet de deux types de lois du travail sur l'activité de grève, à savoir celles visant à réduire telle activité et d'autres qui ne cherchent pas à la réduire mais qui peuvent l'affecter indirectement par leur incidence sur les rapports de force syndical-patronal.

L'activité de grève a fait l'objet de nombreuses études autant théoriques qu'empiriques. Les questions que les économistes se sont posées sur le sujet vont de l'explication de la décision de grève, à celles des variations temporelles, industrielles et internationales de l'activité de grève. Par ailleurs, le cadre légal dans lequel les conventions collectives sont négociées et signées est généralement supposé donné et constant ${ }^{1}$. Pourtant, les lois du travail sont continuellement modifiées, la jurisprudence évolue sans cesse et de nouvelles lois sont régulièrement adoptées. Ces changements au cadre juridique peuvent-ils avoir une incidence sur l'activité de grève?

Gunderson et al. (1986) ont été les premiers chercheurs non seulement à s'intéresser à l'impact des lois du travail sur l'activité de grève mais aussi à tenter d'en évaluer empiriquement les effets. Ils expliquent le recours à la grève, dans le cadre de la négociation d'un contrat de travail, par le coût de

* LACROIX, R., professeur et directeur du Centre de recherche et développement en économique de l'Université de Montréal.

LESPÉRANCE, A., assistant de recherche au Centre de recherche et développement en économique de l'Université de Montréal.

** Nous remercions A. Hollander, C. Montmarquette, A. Raynauld et F. Vaillancourt pour leurs commentaires sur une première version de cet article. Cette recherche a été financée par une subvention du fonds F.C.A.R.

1 Un échantillon représentatif d'écrits récents sur le sujet retiendrait entre autres les articles suivants: COUSINEAU et LACROIX (1986), DUSSAULT et LACROIX (1980), HAYES (1984), KAUFMAN (1982), MAURO (1982), REDER et NEUMANN (1980), SIEBERT et ADDISON (1981), SWIDINSKY et VANDERKAMP (1982) et TRACY (1986). 
celle-ci par rapport au coût de mécanismes alternatifs permettant d'obtenir l'information nécessaire à l'évolution des négociations vers une entente. Selon ces auteurs, étant donné l'imperfection de l'information divulguée, les parties ont recours à des mécanismes capables de générer de l'information ou d'en augmenter la qualité. Ils notent également qu'il existe, outre la grève, de multiples mécanismes institutionnels permettant d'échanger de l'information. C'est ainsi que la constitution de comités conjoints, de procédures de négociation continue, de conciliation, d'arbitrage volontaire ainsi que de règlement des griefs constituent des mécanismes alternatifs à la négociation conventionnelle et à la grève qui lui est occasionnellement associée. Cependant, à l'instar de la grève, ces procédés sont coûteux en temps et en ressources. Dès lors, on peut postuler que plus le coût de ces mécanismes alternatifs est inférieur au coût conjoint de la grève pour les deux parties, pour une quantité d'information donnée, plus l'activité de grève sera réduite. Ainsi, selon ces auteurs, l'adoption de lois du travail créant des mécanismes alternatifs nouveaux pourra avoir une incidence sur l'activité de grève.

Tout en reconnaissant la valeur de ce travail de pionnier, trois raisons principales nous ont incités à remettre en cause l'étude précitée.

D'abord, les auteurs ont confondu deux types de lois du travail: celles qui ne modifient pas les rapports de force entre les parties mais cherchent à réduire le coût de leur évaluation par les parties et celles qui changent les rapports de force. Ces deux types d'interventions législatives ont des effets fort différents sur l'activité de grève, comme nous le préciserons plus loin.

Ensuite, l'évaluation que font les auteurs de l'efficacité de certaines lois pour réduire le coût d'acquisition d'information par les parties est souvent contestable. Nous élaborerons sur ce dernier point en prenant pour exemple la conciliation obligatoire. Les auteurs affirment que la conciliation obligatoire permet de réduire la fréquence des grèves en révélant de l'information. Selon eux, le coût de la grève serait supérieur à celui de la conciliation car celle-ci est subventionnée par le gouvernement. Il importe de préciser que c'est l'aspect obligatoire de la conciliation dont il s'agit. En effet, dans toutes les provinces concernées, la conciliation a toujours joué un rôle important dans la négociation et cela en dépit du fait que certaines législatures l'aient rendue facultative. Ainsi, au sens même des hypothèses développées par Gunderson et al. (1986), le coût de la grève par rapport à la conciliation ne devrait pas être fonction du caractère obligatoire de cette dernière étant donné que l'on suppose que les parties choisissent librement et rationnellement un mécanisme générateur d'information. 
Enfin, si la distinction que nous avons faite entre les deux types de lois du travail est pertinente, le meilleur modèle pour anticiper l'effet de diverses lois du travail sur l'activité de grève devient le «modèle d'accident» de la grève conçu par Siebert et Addison (1981) et développé par Cousineau et Lacroix (1986).

Dans le présent article, nous voulons évaluer l'effet des deux types de lois sur l'activité de grève. Dans une première partie, nous présentons succinctement le «modèle d'accident» de la grève et nous en inférons des attentes quant à l'effet de cinq lois du travail ayant été promulguées à un moment donné dans l'une, l'autre ou les trois provinces canadiennes suivantes: Colombie-Britannique, Québec et Ontario. Une spécification d'une équation de grève intégrant des variables de manière à tenir compte des effets possibles de nouvelles lois du travail est présentée dans la partie suivante et les résultats d'estimations, faites sur la base de données individuelles (conventions collectives) canadiennes, y sont analysés. Enfin, nous tirons les conclusions de cette recherche dans la dernière partie.

\section{LE «MODÈLE D'ACCIDENT» DE LA GRÈVE ET LES LOIS DU TRAVAIL}

Le principe de base du «modèle d'accident» de la grève est que les parties ont toujours intérêt à négocier ${ }^{2}$. La négociation constitue un processus d'échange d'informations permettant de mieux évaluer les rapports de force ainsi que la capacité de payer de la partie patronale. En augmentant l'information disponible aux deux parties, les négociations réduisent les erreurs d'interprétation des rapports de force et de la capacité de payer. Or, ce sont précisément ces erreurs qui conduisent à la grève.

Si les négociations n'étaient pas limitées dans le temps, la probabilité de grève tendrait vers zéro. Malheureusement, un certain nombre de facteurs empêchent une durée illimitée des négociations. D'abord, les informations nécessaires à l'établissement des salaires et des autres conditions de travail qui prévaudront durant la convention collective suivante (i.e. les deux ou trois prochaines années) deviennent obsolescentes tellement rapidement que les négociations sur les points importants du nouveau contrat de travail ne pourront commencer que quelques mois au moins avant la fin du contrat de travail en vigueur. Ensuite, les négociations peuvent difficilement s'éterniser après l'échéance d'une convention collective. En effet, lorsqu'une convention collective est échue, les conditions de travail sont "gelées», c'est-àdire qu'elles n'évoluent plus au même rythme que le coût de la vie. Il y a

2 Pour une présentation complète du modèle et ses développements empiriques, on se référera à SIEBERT et ADDISON (1981) et COUSINEAU et LACROIX (1986). 
donc un coût d'attente pour les travailleurs qui augmente avec le taux d'inflation. De plus, lorsqu'une convention collective s'achève ou est échue, l'incertitude quant aux futures conditions de travail et l'éventualité d'un arrêt de travail empêchent les travailleurs de prendre un ensemble de décisions de consommation et d'investissement. C'est aussi le coût du temps passé à négocier. Enfin, il y a un ensemble de coûts directs de la négociation provenant surtout de frais salariaux encourus par l'équipe de négociation syndicale.

À peu de choses près, les mêmes facteurs expliquent pourquoi l'employeur ne peut lui non plus se permettre une négociation de longue durée. Lui aussi subit un ensemble de coûts directs et indirects reliés à la négociation qui bornent l'espace temporel des négociations.

En résumé, les parties ont intérêt à négocier, d'une part, parce que les négociations sont un moyen efficace de leur éviter une grève coûteuse et, d'autre part, parce qu'elles permettent aux travailleurs d'obtenir des conditions de travail supérieures aux offres initiales de l'employeur. En même temps, elle permet à ce dernier d'obtenir une convention collective moins onéreuse que les demandes initiales des travailleurs. Donc, tous gagnent à négocier. Cependant, compte tenu des coûts de la négociation, les parties doivent choisir une durée optimale de négociation. Cette durée sera celle qui égalisera les bénéfices marginaux de la négociation à ses coûts marginaux ${ }^{3}$.

En optimisant la durée de négociation, les parties acceptent une probabilité de grève non nulle. Une telle décision est semblable à celle que prend l'individu lorsqu'il décide du temps qu'il mettra pour effectuer un voyage en auto. En effet, compte tenu de la valeur de son temps, l'individu décide de rouler à une certaine vitesse. À cette vitesse, sur la distance à faire, il sait qu'il choisit implicitement une probabilité d'accident qui sera d'autant plus forte que la vitesse retenue est élevée. L'accident, s'il se produit, sera toujours le résultat d'une erreur de sa part ou de celle des autres conducteurs.

Nous avons une situation similaire pour la grève qui est le résultat d'une erreur, d'un accident de négociation. Contrairement à l'approche adoptée par Gunderson et al. (1986), les parties ne choisissent pas au départ la grève parmi un ensemble de moyens alternatifs d'obtenir l'information mais acceptent plutôt une certaine probabilité de grève en choisissant un temps de négociation qui en maximise le bénéfice net.

3 Nous aurons la même durée optimale pour les deux parties puisque, s'il advient qu'une des parties ait intérêt à négocier plus longtemps que l'autre, elle pourra toujours inciter l'autre partie à continuer la négociation en concédant une partie des avantages qu'elle escompte d'une négociation plus longue. Ce faisant, le temps optimal de négociation sera raccourci pour la partie qui concède et allongé pour celle qui bénéficie de la concession. On tendra ainsi à un temps identique pour les deux parties. 
Un ensemble de facteurs, modifiant la qualité ou la quantité des informations requises pour évaluer les rapports de force et la capacité de payer de l'entreprise, peuvent affecter autant la localisation de cette relation d'arbitrage entre la durée de négociation et la probabilité de grève que le choix de la durée optimale de négociation. Comme l'ont montré Cousineau et Lacroix (1986) et Lacroix (1987), les variations dans ces facteurs peuvent très bien expliquer les variations temporelles, interindustrielles et internationales (interrégionales) dans l'activité de grève. L'avènement de nouvelles lois du travail ou la modification de celles existantes, si elles changent la qualité ou la quantité d'informations utiles aux parties, auront un effet sur l'activité de grève.

Comme nous l'avons dit antérieurement, eu égard à leur impact sur l'activité de grève, nous pouvons classer les nouvelles lois du travail (ou les modifications aux anciennes lois) en deux catégories. Il y a celles qui visent implicitement la réduction de l'activité de grève et les autres qui pourront indirectement affecter l'activité de grève parce qu'elles modifient les rapports de force entre les parties. Nous allons examiner successivement ces deux types de lois et préciser nos attentes face à celles que nous avons retenues pour notre étude empirique.

Dans le cadre du «modèle d'accident» de la grève, les nouvelles lois (ou modifications de lois) introduites pour réduire l'activité de grève atteindront le but visé si elles ont l'un, l'autre ou les deux effets suivants: elles augmentent la rapidité de transmission à l'autre partie d'informations détenues en exclusivité par chacune des parties; elles accroissent la quantité d'informations détenues en exclusivité qui sont transmises entre les parties. L'examen de l'évolution des lois du travail dans trois provinces canadiennes (Colombie-Britannique, Québec et Ontario) au cours de la période 1967-1982 nous a amenés à retenir trois types de lois (ou modifications à des lois existantes) qui furent incontestablement introduites pour réduire l'activité de grève. Il s'agit des lois portant sur le caractère obligatoire de la conciliation, des lois relatives aux délais de négociation et, enfin, des lois portant sur le vote de grève.

\section{La conciliation obligatoire}

Dans toutes les provinces canadiennes, la conciliation constitue la partie charnière autour de laquelle s'articule le processus de négociation. En effet, les législateurs ont longtemps cru que la conciliation suffisait pour désamorcer des conflits de travail et ont voté des lois qui prohibaient l'exercice du droit de grève sans l'intervention préalable d'un conciliateur. Le caractère obligatoire de la conciliation retardait ainsi l'exercice du droit de grève. 
En 1968 et 1978 respectivement, les gouvernements de la ColombieBritannique et du Québec se sont rendus aux arguments de certains praticiens qui prétendaient que le caractère obligatoire de la conciliation nuisait à la bonne marche des négociations ou en retardait le processus. Dans ces provinces, la conciliation cessait d'être obligatoire, chacune des parties conservant toutefois le droit d'exiger la présence d'un conciliateur au moment jugé opportun ${ }^{4}$.

On doit maintenant se demander si la présence d'un conciliateur dans le cadre des négociations peut modifier la rapidité de transmission d'informations exclusives à l'une ou l'autre des parties et la quantité d'informations transmises. Selon le «modèle d'accident» de la grève, les parties choisissent une période optimale de négociation qui est celle qui égalise le coût marginal de la négociation avec son bénéfice marginal. La conciliation obligatoire étant perçue par les parties comme une étape nécessaire avant la grève, cette période de temps sera intégrée au calcul des parties mais ne devrait pas changer la période optimale de négociation. En effet, les parties peuvent toujours faire varier la période de négociations précédant la fin de la convention collective. Et si la période optimale des négociations n'est pas modifiée, toutes autres choses étant égales par ailleurs, la probabilité de grève ne sera pas affectée par une telle mesure législative.

Évidemment, toutes choses ne seront pas égales par ailleurs si la conciliation donne lieu à l'échange d'informations entre les parties qui ne l'auraient pas été autrement. Dans un contexte de conciliation obligatoire, il se pourrait que pour des fins stratégiques, les parties attendent la conciliation pour transmettre des informations qui leur sont exclusives. On ne peut cependant en déduire que ces informations n'auraient pas été transmises en l'absence de conciliation. Au contraire, elles l'auraient peut-être été plus rapidement. En somme, la conciliation ne modifie ni la qualité ni la quantité d'informations détenues en exclusivité par chacune des parties. De plus, elle ne change pas l'intérêt des parties à échanger ces informations. Elle ne fait que déplacer le moment où des informations détenues par les parties seront échangées.

Dans ces conditions, ni l'adoption d'une loi rendant obligatoire la conciliation avant la grève ni l'abolition d'une telle loi ne devraient avoir d'incidence sur l'activité de grève. Notons que Gunderson et al. (1986) arrivaient quant à eux à anticiper un effet négatif de la conciliation obligatoire sur l'activité de grève.

4 On trouvera le détail de ces modifications pour le Québec, à l'article 36 de la Loi modifiant le Code du travail et la Loi du ministère du Travail et de la Main-d'oeuvre, L.Q. 1977, chap. 41, modifiant les articles 41 à 46 du Code du travail et pour la Colombie-Britannique, à l'article 25 de An Act Respecting Collective Bargaining and Mediation, S.B.C. 1968, chap. 26. 


\section{Délai de négociation et avis}

Les lois régissant les relations du travail dans les trois provinces canadiennes retenues prévoient qu'une des deux parties peut, avant l'expiration de la convention collective, forcer l'autre partie à commencer la négociation. Il suffit d'expédier un avis avec la date et le lieu des négociations. Au cours de la période 1969-1981, les trois provinces étudiées ont modifié ces délais. En Ontario et en Colombie-Britannique, la période est passée de 3 à 4 mois alors qu'au Québec, elle est passée de 2 à 3 mois. D'après le modèle présenté, les parties ayant implicitement ou explicitement convenu d'une période de négociation qui leur paraissait optimale compte tenu des avantages et des coûts de la négociation, l'intervention du législateur sur les délais de négociation, en ne modifiant ni les avantages ni les coûts des négociations, ne devrait pas affecter les choix des parties et donc la probabilité de grève. En fait, tout ce qu'une telle loi occasionnera c'est une différence entre la durée nominale et la durée réelle des négociations. La durée nominale est fonction de la date de l'avis envoyé par l'une des parties alors que la durée réelle, c'est-à-dire la période de temps durant laquelle il y a vraiment des négociations, dépendra du choix optimal fait par les parties.

\section{Les modalités du vote de grève}

Les provinces canadiennes ont cru nécessaire d'établir des règles lors de la tenue d'un vote de grèves. D'une manière générale, les lois exigent que ces consultations soient faites au scrutin secret. En 1972, l'Ontario demandait que les procédures de vote soient telles que les employés autorisés à voter aient vraiment l'opportunité d'exprimer leur choix. En 1980, cette même province permettait à l'employeur d'exiger que ses employés se prononcent personnellement sur ses offres.

Le Québec allait dans le même sens en 1978 en exigeant qu'un avis de 48 heures annonçant la tenue d'un vote de grève soit expédié aux membres du syndicat. La même année, la Colombie-Britannique promulguait également un règlement prévoyant une procédure exhaustive du déroulement du vote de grève. Celui-ci comprenait l'obligation de donner l'occasion raisonnable aux salariés de voter.

Ces mesures contribuent d'une manière générale à affirmer le caractère démocratique de l'exercice du droit de grève. Toutefois, selon le modèle de grève que nous avons présenté, la partie syndicale a un intérêt certain à ce que l'information sur l'intensité de l'appui de la base syndicale aux revendications faites par les dirigeants syndicaux soit non seulement révélée mais 
crédible. En effet, la menace de grève constitue une composante majeure du rapport de force et le vote de grève rend cette menace crédible. La partie syndicale a donc intérêt à adopter une procédure de vote qui donne à la menace de grève le plus de crédibilité possible. L'intervention du législateur ne devrait pas modifier le comportement rationnel des agents négociants.

En résumé, ces modifications législatives portant sur le vote de grève ne changent pas la qualité de l'information fournie à l'employeur quant à l'intensité de la menace de grève et ne devraient pas modifier la probabilité de grève par ce biais. Ici encore, notre approche nous conduit à avoir des attentes différentes de celles de Gunderson et al (1986) qui escomptaient une incidence négative sur l'activité de grève de ces lois.

À l'aide du «modèle d'accident» de la grève, on arrive donc à la conclusion que trois lois qui visaient une réduction de l'activité de grève ne devraient pas atteindre cet objectif. On vérifiera empiriquement cette première conclusion dans une prochaine section.

Comme nous l'avons souligné antérieurement, de nouvelles lois du travail qui n'ont pas été introduites pour réduire l'activité de grève ont pu avoir une incidence sur cette dernière par l'effet qu'elles ont sur l'es rapports de force entre syndicats et employeurs. Une nouvelle loi du travail modifiant les rapports de force aura ultimement un effet sur les salaires, effet positif si la modification est à la faveur des syndicats. Ce changement n'affectera pas à long terme l'activité de grève dans la mesure où l'état des nouveaux rapports de force est connu des deux parties. Toutefois, il y aura une période d'incertitude avant que l'impact de la nouvelle loi sur les rapports de force soit proprement évalué. En d'autres mots, l'information sur les rapports de force est temporairement perturbée par l'avènement d'une nouvelle loi du travail. Selon le «modèle d'accident» de la grève, cette perturbation de l'information aura un effet positif sur la probabilité de grève. Il faut, cependant, remarquer que cette perturbation de l'information ne durera que le temps requis pour que les parties réévaluent leurs nouveaux rapports de force. Dès lors, ces nouvelles lois n'auront qu'un effet temporaire sur l'activité de grève, effet qui s'atténuera pour éventuellement disparaître. Seul l'effet sur les salaires persistera.

Il nous a paru qu'au cours de la période 1967-1982, deux nouvelles lois du travail avaient toutes les caractéristiques de nouvelles lois non spécifiquement introduites pour réduire l'activité de grève mais modifiant de façon significative les rapports de force. Il s'agit des dispositions sur le piquetage que l'on trouve dans la refonte de 1974 du Labour Relations Act de la Colombie-Britannique et des dispositions législatives anti-briseurs de grève promulguées en 1978 par le gouvernement du Québec. 
Piquetage secondaire et loi anti-briseurs de grève

En Colombie-Britannique, c'est en 1974 que le parlement accordait au tribunal chargé d'appliquer le Labour Relations Act une juridiction exclusive sur les matières relatives au piquetage. La Cour supérieure perdait du même coup compétence sur un sujet qui lui avait été jusqu'alors réservé. La refonte de 1974, non seulement reconnaissait le droit de piqueter, mais protégeait par une immunité absolue ceux qui s'en prévalaient. De plus, la législation alors promulguée légalisait les moyens d'action de type secondaire, ${ }^{5}$ tels le piquetage et le boycottage dirigés contre une tierce partie non directement engagée dans le conflit. Les moyens de pression secondaires peuvent s'avérer, dans certains cas, plus efficaces que des actions directement dirigées contre la partie patronale ${ }^{6}$.

Le 26 juin 1975, le gouvernement de la Colombie-Britannique introduisait de nouvelles modifications législatives ${ }^{7}$ exigeant que la partie patronale obtienne, préalablement à l'institution de poursuite civile contre la partie syndicale, l'autorisation du tribunal administratif chargé d'appliquer les lois du travail.

Les textes de 1974 et 1975 occasionnaient un bouleversement du pouvoir des syndicats. Cependant, l'importance de ce changement demeurait incertaine. On pouvait se demander, par exemple, dans quelle optique le tribunal nouvellement institué assumerait ses fonctions de régulateur du droit de piqueter? À court terme, les parties étaient donc incapables de mesurer l'ampleur du changement intervenu dans leurs rapports de force. Ainsi, la qualité de l'information permettant aux parties d'estimer leur pouvoir de négociation s'étant temporairement détériorée, on pouvait s'attendre à une augmentation de l'activité de grève, augmentation qui se résorberait à mesure que l'effet des nouvelles dispositions législatives sur les rapports de force se préciserait.

Le premier février 1978, le parlement du Québec promulguait des dispositions législatives anti-briseurs de grève. Aux termes de ces dispositions, on interdisait à l'employeur d'utiliser les services de personnes pour remplir les fonctions de salariés ayant légalement déclaré la grève ou ayant été «lock-outés». Cette règle souffrait, toutefois, une double exception. Ainsi, le travail normalement réservé aux salariés en grève ou en lock-out pouvait être effectué en partie, soit par les cadres de l'entreprise, soit par des salariés

5 L'arrêt Herses of Woodstock v. Goldstein, (1963) 38 D.L.R. (2d) 449, avait jugé ces actions illégales.

6 Voir à ce sujet H.W. ARTHURS et al. (1984), p. 250 et J.M. WEILER (1984), pp. 177-178.

7 S.B.C. 1975 , chap. 33 , art. 8. 
du même établissement de l'employeur mais n'appartenant pas à l'unité de négociation en grève ou en lock-out. Dans les deux cas, cette règle d'exception exigeait que les personnes utilisées à des fins de remplacement aient été embauchées avant le début de la phase de négociation.

Donc le Québec, comme la Colombie-Britannique, modifiait l'équilibre des forces en présence. Toutefois, plutôt que d'accroître directement l'efficacité des moyens de pression à la disposition des syndicats, il imposait une hausse du coût de la grève à l'employeur. Nous nous attendons à ce que ce changement dans les rapports de force ait eu un effet positif et temporaire sur l'activité de grève, parce qu'il a introduit une incertitude passagère quant à l'importance du pouvoir syndical de négociation. Encore une fois, nos attentes quant à l'effet de cette loi sur l'activité de grève sont complètement différentes de celles de Gunderson et al. (1986) qui étaient incertains de son effet et ne concluaient pas au caractère temporaire de l'effet en cause.

\section{LE MODÈLE ET LES RÉSULTATS EMPIRIQUES}

\section{Le modèle}

L'équation de grève que nous avons retenue pour estimer l'impact des diverses lois sur l'activité de grève est, à quelques petites modifications près, celle de Cousineau et Lacroix (1986). Cette équation avait été spécifiée à partir du modèle d'accident de la grève développé par Siebert et Addison (1981) et cherchait à expliquer les variations intertemporelles et interindustrielles de l'activité de grève.

Les variables retenues par les auteurs pour expliquer les variations intertemporelles de l'activité de grève, et que nous reprenons ici, sont au nombre de cinq. Il s'agit d'abord de trois indicateurs pouvant être utilisés par les parties pour évaluer les rapports de force et la capacité de payer de l'entreprise, à savoir le taux d'utilisation de la capacité du secteur industriel, l'inflation anticipée et un indice du taux de vacances. Dans le cadre du modèle d'accidents de la grève, ce n'est pas l'évolution du rapport de force qui occasionne la grève mais bien le degré d'incertitude quant à cette évolution. C'est donc sous la forme des coefficients de variation que furent intégrés à l'équation de grève les trois indicateurs précités, à savoir (TUCC), ${ }^{8}$ le coefficient de variation du taux d'utilisation de la capacité au niveau de l'industrie; (INFLC) le coefficient de variation de l'inflation anticipée; et

8 Une définition précise et les sources statistiques de toutes les variables sont disponibles sur demande auprès des auteurs. 
(ITVC), le coefficient de variation de l'indice de taux de postes vacants. Deux autres variables ont été retenues pour expliquer les variations intertemporelles de l'activité de grève. Il s'agit d'abord de l'inflation au moment de la négociation du contrat de travail (INFLA). Cette variable cherche à capter le coût de la négociation qui augmente avec l'inflation au moment des négociations puisque, lorsqu'un contrat est échu, l'ensemble des conditions de travail est figé. Dans ces conditions, plus les taux d'inflation sont élevés, plus les conditions de travail réelles se détérioreront rapidement. Nous avons ajouté à notre modèle une variable (CONTR) prenant une valeur croissante durant la période de contrôle des revenus et des prix (octobre 1975 à avril 1978). Étant donné que ces contrôles circonscrivaient de plus en plus étroitement les ententes salariales possibles, nous avons supposé que l'activité de grève serait graduellement réduite pendant le programme de contrôle.

Trois variables explicatives des variations interindustrielles ou interfirmes de l'activité de grève ont été retenues. Il s'agit, d'abord, du degré d'exposition à la concurrence internationale d'une industrie (ABREX). La logique sous-jacente à cette variable est la suivante: plus une entreprise est exposée à la concurrence internationale, plus la quantité d'informations requises pour évaluer les rapports de force et la capacité de payer de l'entreprise est considérable. Il faut, en effet, tenir compte de l'évolution possible des marchés étrangers, des taux de changes, des coûts de transports, etc. Selon cette approche, les risques d'erreurs d'évaluation sont alors plus grands et la probabilité de grève devrait être plus considérable dans ces secteurs davantage exposés. Toutefois, s'il advient que les industries exposées à la concurrence internationale soient aussi celles où, dans l'ensemble, la concurrence est la plus vive, on pourrait avoir une atténuation et possiblement un renversement du premier effet décrit. En effet, les coûts de la grève pour les deux parties étant généralement plus élevés dans les industries où la concurrence est forte, les parties auront intérêt à développer des protocoles de négociation réduisant la probabilité de grève (Reder et Neumann, 1980).

Comme deuxième variable, nous avons retenu le nombre d'employés couverts par la convention collective (NEMPL). Nous nous attendons à ce que l'activité de grève soit plus considérable lorsque le nombre d'employés est grand. Deux raisons expliqueraient cette relation. D'abord, l'augmentation du nombre d'employés s'accompagnera généralement d'une plus grande hétérogénéité des caractéristiques et des exigences de la main-d'oeuvre impliquant des conventions collectives beaucoup plus complexes, plus difficiles à négocier et plus susceptibles de connaître des accidents de négociation résultant en une grève. Ensuite, le grand nombre d'em- 
ployés augmente les difficultés de communication entre les dirigeants syndicaux et la base syndicale, ce qui peut occasionner des erreurs de perception quant à l'intensité des revendications syndicales.

La dernière variable pouvant expliquer des différences interfirmes de l'activité de grève à un moment donné du temps est la durée du contrat antérieur à celui en négociation (LDUREE). Plus le contrat antérieur aura été long, plus les griefs accumulés pourront être nombreux et les déceptions importantes par rapport aux attentes initiales. Pour un temps de négociation donné, la probabilité de grève devrait s'accroître avec la durée du contrat antérieur.

Il nous faut maintenant ajouter à ce modèle de grève des variables pour tenir compte de l'incidence possible de nouvelles lois du travail sur l'activité de grève. Deux des trois lois ayant possiblement une incidence directe sur l'activité de grève, à savoir le caractère obligatoire de la conciliation (CON) et les modalités des votes de grève (VOT), ont été ajoutées au modèle sous forme de variables dichotomiques prenant la valeur 1 lorsqu'elles sont en vigueur dans l'une des trois provinces et 0 autrement. Pour ce qui est de la variable délais de négociation (NEG), nous avons retenu le nombre de mois légalement requis et qui a changé dans chacune des provinces au cours de la période comme nous l'avons souligné précédemment. Pour les deux lois affectant indirectement l'activité de grève par leur incidence sur les rapports de force entre syndicats et employeurs, une variable dichotomique (RP) a été ajoutée au modèle de grève pour en tenir compte. Cependant, la période de temps durant laquelle cette variable dichotomique prend la valeur 1 , lorsque l'une et l'autre lois furent introduites, a été déterminée empiriquement. Nous avons postulé au départ, pour les raisons données antérieurement, que l'effet de ces lois sur l'activité de grève est temporaire. Encore faut-il estimer cette période de temps, ce que nous faisons, en prenant comme critère de choix la période pour laquelle la variable était statistiquement la plus significative. Dans le cas de la loi anti-briseurs de grève, la période retenue fut de 15 mois, alors qu'elle fut de 22 mois dans le cas des changements législatifs de la Colombie-Britannique.

\section{Les résultats}

Les données que nous avons utilisées pour estimer notre modèle de grève ont été tirées de la banque de données de Travail Canada sur les conventions collectives regroupant 500 travailleurs ou plus. Nous avons retenu les 1272 conventions collectives du secteur manufacturier signées entre le $1^{\text {er }}$ janvier 1969 et le 31 décembre 1981 dans les provinces de Québec, de l'Onta- 
rio et de la Colombie-Britannique. De ces 1272 contrats de travail, 284 furent signés après une grève, soit $22,3 \%$. Compte tenu de la nature dichotomique de notre variable dépendante (égale à 1 si le contrat de travail a été signé après une grève et égale à 0 si le contrat a été signé sans grève), nous avons utilisé une procédure d'estimation Probit. Les résultats des estimations sont présentés au tableau 1. Pour pouvoir interpréter ces résultats en terme de variations de probabilités de grève, nous présentons dans le même tableau les coefficients transformés. Dans le cas des variables continues, les coefficients transformés correspondent à l'accroissement de la probabilité de grève résultant du passage de la valeur de la variable de sa moyenne à cette dernière plus un écart-type. Pour les variables dichotomiques, il s'agit de la variation dans la probabilité de grève lorsque la variable passe d'une valeur 0 à une valeur 1 .

Les coefficients de toutes les variables explicatives autres que les variables légales ont le signe attendu et, à l'exception de la variable retenue pour estimer l'effet de la politique de contrôle des prix et des revenus (CONTR), toutes sont statistiquement significatives (modèle 1 du tableau 1). Ainsi, le fait qu'une entreprise appartienne à un secteur industriel exposé à la concurrence internationale augmente la probabilité de grève de 11 points de pourcentage. Si le nombre d'employés dans une entreprise passe de 1343 (dimension moyenne des établissements dans notre échantillon) à 2186 , la probabilité de grève s'accroît de 3 points de pourcentage. De même, si la durée de la convention antérieure d'une entreprise passe de 26,3 mois à 34,8 mois, la probabilité de grève croîtra de 8 points de pourcentage. À l'aide des valeurs moyennes et des écarts-types des variables données en annexe, on peut interpréter de la même façon l'effet des variables autres que légales.

Les coefficients des quatre variables légales (CON, NEG, VOT, RP) sont aussi conformes aux attentes que nous avions formulées à partir du modèle d'accident de la grève. En effet, nous avions conclu que la promulgation ou le retrait de lois sur la conciliation obligatoire, les délais de négociation et les modalités du vote de grève n'auraient aucun effet sur l'activité de grève. Or, aucun des trois coefficients des variables en cause n'est statistiquement significatif. Par contre, le coefficient de la variable RP nous indique que les deux lois qui ont modifié les rapports de force ont eu un impact temporaire mais très important sur l'activité de grève en faisant augmenter la probabilité de grève de 19 points de pourcentage.

Il nous faut maintenant appuyer notre affirmation voulant que les lois modifiant les rapports de force n'avaient qu'un effet temporaire sur la probabilité de grève. Pour ce faire, nous avons tout simplement ajouté une période additionnelle de 18 mois à la variable dichotomique RP et réestimé 
le modèle ${ }^{9}$. Nous constatons que les résultats que nous avions trouvés pour les variables autres que RP sont relativement stables (modèle 2, tableau 1). Par contre, pour la variable RP, l'allongement de la période de 18 mois fait passer le coefficient transformé de 0,189 à 0,065 et la statistique t passe de 3,255 à 1,364 . Sans que nous puissions être tout à fait certains qu'un accroissement additionnel de la durée aurait fait disparaître RP comme variable explicative de la probabilité de grève, la tendance que notre expérience nous a permis de déceler va en ce sens. En somme, les résultats qui précèdent appuieraient l'hypothèse selon laquelle l'introduction de lois du travail changeant les rapports de force entre syndiqués et employeurs n'aurait qu'un effet temporaire sur l'activité de grève.

\section{CONCLUSION}

L'introduction de nouvelles lois a incontestablement un effet soit temporaire soit permanent sur l'activité de grève. Nous avons montré qu'un modèle de grève était nécessaire pour pouvoir prévoir cet effet. Nous avons aussi montré comment le «modèle d'accident» de la grève conduisait à des inférences contredisant celles qu'on aurait pu tirer de toutes analyses superficielles et non guidées par un modèle de grève éprouvé.

Nos résultats empiriques concernant l'incidence sur l'activité de grève du caractère obligatoire de la conciliation, des modalités du vote de grève et des délais de négociation appuient les hypothèses que nous avions faites à partir de notre modèle de grève, à savoir qu'aucune de ces lois ne devait avoir d'effet sur l'activité de grève. Par contre, nous avons trouvé que la loi sur le piquetage secondaire ainsi que la loi anti-briseurs de grève ont eu un effet positî́ temporaire mais néanmoins important sur l'activité de grève. C'est aussi ce que nous avait fait anticiper notre modèle de grève.

Ces résultats montrent qu'il est possible, à l'aide d'un modèle économique de la grève, de prévoir les effets de nouvelles lois du travail sur l'activité de grève. Cette approche à l'étude des législations du travail pourrait s'avérer fort utile dans l'avenir.

9 Ces 18 mois étaient l'allongement maximum que notre période échantillonnale nous permettait puisque la loi anti-briseurs a été adoptée au Québec en 1978 et notre période échantillonnale se termine en décembre 1981. Par ailleurs, nous avons estimé une équation où étaient distingués les effets de RP au Québec et en Colombie-Britannique. Nous avons alors trouvé un impact significatif de RP sur les probabilités de grève de $19,1 \%$ en Colombie-Britannique et de $15,8 \%$ au Québec. 
Tableau 1

\section{Résultats empiriques}

MODELLE I

\begin{tabular}{|c|c|c|}
\hline Variables & Coefficients & $\begin{array}{l}\text { Coefficients } \\
\text { transformés }\end{array}$ \\
\hline $\begin{array}{l}\text { ABREX } \\
\mathrm{t}=\end{array}$ & $\begin{array}{l}0,427 \\
(4,340)\end{array}$ & 0,112 \\
\hline NEMPL & $\begin{array}{l}0,00004 \\
(2,675)\end{array}$ & 0,010 \\
\hline LDUREE & $\begin{array}{c}0,032 \\
(5,812)\end{array}$ & 0,084 \\
\hline INFLC & $\begin{array}{l}10,455 \\
(2,411)\end{array}$ & 0,042 \\
\hline TUCC & $\begin{array}{c}8,856 \\
(2,037)\end{array}$ & 0,025 \\
\hline ITVC & $\begin{array}{c}4,020 \\
(1,410)\end{array}$ & 0,021 \\
\hline CONTR & $\begin{array}{c}-0,016 \\
(-0,134)\end{array}$ & $-0,004$ \\
\hline INFL & $\begin{array}{c}0,087 \\
(3,400)\end{array}$ & 0,058 \\
\hline $\mathrm{RP}$ & $\begin{array}{c}0,574 \\
(3,255)\end{array}$ & 0,189 \\
\hline $\mathrm{CON}$ & $\begin{array}{c}0,033 \\
(0,269)\end{array}$ & 0,009 \\
\hline NEG & $\begin{array}{c}0,003 \\
(0,037)\end{array}$ & 0,001 \\
\hline VOT & $\begin{array}{c}-0,004 \\
(-0,307)\end{array}$ & $-0,012$ \\
\hline Constant & $\begin{array}{l}-3,339 \\
(-8,336)\end{array}$ & \\
\hline Pseudo $\mathrm{R}^{2}$ & 0,159 & \\
\hline $\begin{array}{l}\text { Ratio de la fonction } \\
\text { de vraisemblance }\end{array}$ & 139,493 & \\
\hline $\begin{array}{l}\text { Nombre } \\
\text { d'observations }\end{array}$ & 1272 & \\
\hline
\end{tabular}

MODELLE 2

Coefficients

Coefficients

transformés

0,420

0,110

$(4,285)$

0,00004

$(2,543)$

0,032

$(5,892)$

9,069

$(2,110)$

9,529

$(2,208)$

5,261

$(1,867)$

0,032

$(0,009)$

0,083

$(3,266)$

0,009

0,085

0,037

0,027

0,028

0,0003

0,056

0,220

0,065

$(1,364)$

$-0,028$

$(-0,203)$

0,007

$(0,089)$

$-0,008$

0,002

$-0,053$

$(-0,372)$

$-0,015$
$-3,281$

$(-8,036)$

0,149

130,841

1272 


\section{BIBLIOGRAPHIE}

ARTHURS, H.W., D.D. CARTER et H.J. GLASBEEK, Labour Law and Industrial Relations in Canada, Butterworths, Toronto, 1984.

COUSINEAU, J.-M. et R. LACROIX, «Imperfect Information and Strikes: An Analysis of Canadian Experiences, 1967-1982», Industrial and Labor Relations Review, vol. 39, no 3, avril 1986, pp. 377-87.

DECALUWÉ, Bernard, «Les branches exposées et abritées de l'économie canadienne: une évaluation empirique», Conseil économique du Canada, document no $163,1980$.

DUSSAULT, F. et R. LACROIX, «Activité de grève: un test des hypothèses explicatives traditionnelles», Canadian Journal of Economics, 13, novembre 1980, pp. 632-44.

GUNDERSON, Morley, J. KERVIN et F. REID, «Logit Estimates of Strike Incidence from Canadian Contract Data», Journal of Labor Economics, vol. 4, no 2, avril 1986, pp. 257-76.

HAYES, Beth, «Unions and Strikes with Asymmetric Information», Journal of Labor Economics, 2, janvier 1984, pp. 57-83.

KAUFMAN, Bruce, «The Determinants of Strikes in the United States, 1900-1977», Industrial and Labor Relations Review, vol. 35, juillet 1982, pp. 473-91.

LACROIX, R., Les grèves au Canada: causes et conséquences, Presses de l'Université de Montréal, Montréal, 1987,

MAURO, Martin J., «Strikes as a Result of Imperfect Information», Industrial and Labor Relations Review, vol. 35, juillet 1982, pp. 522-38.

REDER, Melvin W. et George R. NEUMANN, «Conflict and Contract: The Case of Strikes», Journal of Political Economy, 88, octobre 1980, pp. 867-86.

SIEBERT, W., Stanley et John T. ADDISON, «Are Strikes Accidental?», Economic Journal, 91, juin 1981, pp. 389-404.

SWIDINSKY, Robert et John VANDERKAMP, «A Micro-Econometric Analysis of Strike Activity in Canada», Journal of Labor Research, 3, automne 1982, pp. 455-72.

TRACY, Joseph S., «An Empirical Test of an Asymmetric Information Model of Strikes», Working Paper no 1870, NBER' mars 1986.

WILER, J.M., The Labour of British Columbia in the 1980's, Carswell Legal Publication, Calgary, 1984. 


\section{New Labor Laws and Strike Activity}

Strike activity has been the subject of numerous theoritical and empirical studies. Questions that economists have tried to answer go from the workers' decision to go on strike to those concerning variations of strike activity over time and across industries and countries. However, the legal framework in which collective agreements are negotiated and signed is generally considered given and constant. But labor laws change over time and new labor laws are regularly voted. Do these modifications in the legal framework have an impact on strike activity?

Gunderson et al's study is the first trying to anticipate the impact of labor laws on strike activity and to evaluate empirically the expected effects. They explain the choice to go on strike during the negotiation of a labor contract by the strike cost versus the cost of alternative processes which could generate the same information needed to reach an agreement. Since each and every means to generate a specific useful information during a contract negotiation is costly, one can assume that if the cost of alternative means is lower than the strike cost, parties will agree to use these alternative means. Thus, the adoption of labor laws giving new ways to generate the same information could have an effect on strike activity.

We had three reasons to question Gunderson et al's article. First, they have mistaken two types of labor laws: those which, without changing the relative bargaining power of the parties, try to reduce the cost of its evaluation by both parties; and those which really change the relative bargaining power. Secondly, the evaluation done by the authors of the efficiency of certain labor laws to reduce the cost to obtain information by both parties is often questionable. Finally, we think that the best model to anticipate the effect of different labor laws on strike activity is Siebert and Addison's «accident model» of strike.

In this article, using Siebert and Addison's strike model, hypotheses are proposed concerning the effects of the following five labor laws promulgated in three canadian provinces (British Columbia, Ontario and Québec) on strike activity: compulsory conciliation, mandatory strike vote, negotiation periods, secondary strike pickets and prohibiting the use of replacement workers. Our empirical results sustain the hypothesis made on the effect of the first three types of labor laws. None of them have a significant effect on strike activity. On the other hand, we find that the secondary picketing possibility and the law prohibiting the case of replacement workers have had an important temporary positive effect on strike activity. We also had anticipated this effect with our strike model. 